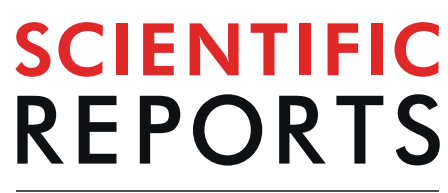

natureresearch

\title{
Comparison between blood pressure during obstructive respiratory events in REM and NREM sleep using pulse transit time
}

\begin{abstract}
Aljohara S. Almeneessier (10) ${ }^{1,2^{*}}$, Mana Alshahrani ${ }^{1}$, Salih Aleissi ${ }^{1}$, Omeima S. Hammad ${ }^{1}$, Awad H. Olaish ${ }^{1}$ \& Ahmed S. BaHammam (10) ${ }^{1,3 *}$

Rapid eye movement-predominant obstructive sleep apnea has been shown to be independently associated with hypertension. This study aimed to non-invasively measure blood pressure during the rapid eye movement (REM) and non-rapid eye movement (NREM) obstructive events and the postobstructive event period. Thirty-two consecutive continuous positive airway pressure-naïve obstructive sleep apnea patients (men, $50 \%$ ) aged $50.2 \pm 12$ years underwent overnight polysomnography. Blood pressure was assessed indirectly using a validated method based on the pulse transit time and pulse wave velocity during the NREM and REM obstructive events (both apneas and hypopneas) and the postobstructive event period. Among the recruited patients, 10 (31.3\%) had hypertension. Mean apneahypopnea index was $40.1 \pm 27.6$ events/hr. Apnea-hypopnea indexes were $38.3 \pm 30.6$ and $51.9 \pm 28.3$ events/hr for NREM and REM sleep, respectively. No differences were detected in obstructive respiratory event duration or degree of desaturation between REM and NREM sleep. Additionally, no difference in blood pressure (systolic and diastolic) was detected between REM and NREM sleep during obstructive events and post-obstructive event period. Simple linear regression identified history of hypertension as a predictor of increased systolic blood pressure during obstructive events and postobstructive event period in both rapid eye movement and non-rapid eye movement sleep. Oxygen desaturation index was also a predictor of increased systolic blood pressure during obstructive events and post-obstructive event period in REM sleep. When obstructive event duration and the degree of desaturation were comparable, no difference in blood pressure was found between REM and NREM sleep during obstructive events and post-obstructive event period.
\end{abstract}

Obstructive sleep apnea (OSA) is a common sleep disorder that occurs during rapid eye movement (REM) and non-REM (NREM) sleep ${ }^{1}$. REM sleep constitutes approximately $25 \%$ of the total sleep time in healthy adults. Interaction of several factors leads to longer duration and greater severity of oxygen desaturation during obstructive events in REM sleep than in NREM sleep. REM-predominant or REM-only OSA occurs in one-third of patients presenting to clinical sleep disorder services ${ }^{2,3}$.

It has been proposed that REM-OSA has more deleterious effects on the cardiometabolic system than does NREM-OSA. During REM sleep, sympathetic activity increases, and cardiovascular instability is prominent $^{4}$. Recent studies suggest that REM-only OSA with an apnea-hypopnea index (AHI) $>15$ events/h or NREM AHI $<5$ events/h is independently associated with prevalent and incident hypertension, as well as with non-dipping of the nocturnal blood pressure $(\mathrm{BP})^{5,6}$. Usually, autonomic functions are stable during NREM sleep; however, significant instability in autonomic functions can be observed during REM sleep ${ }^{7}$. The baroreflex gain

${ }^{1}$ University Sleep Disorders Center, College of Medicine, King Saud University, Riyadh, Saudi Arabia. ${ }^{2}$ Family and Community Medicine, College of Medicine, King Saud University, Riyadh, Saudi Arabia. ${ }^{3}$ Strategic Technologies Program of the National Plan for Sciences and Technology and Innovation in the Kingdom of Saudi Arabia, Riyadh, Saudi Arabia. *email: Aljoharas@yahoo.com; ashammam2@gmail.com 
is heightened in response to BP increments during NREM sleep, and the increased baroreflex gain probably helps to maintain stable low BP during NREM sleep ${ }^{7}$. On the other hand, the instability in autonomic functions during REM sleep produces an imbalance between parasympathetic and sympathetic influences, which results in sudden and abrupt changes in $\mathrm{BP}^{7}$.

An earlier study found that the immediate post-apnea period was associated with increased mean arterial pressure, which was preceded by a rise of muscle sympathetic nerve activity ${ }^{8}$ however, none of the studied patients had REM sleep. In addition, an association of rapid oscillations in BP with apneic events has been observed during sleep in OSA patients ${ }^{9-16}$. Mean BP decreases during normal sleep but can increase during sleep in OSA patients ${ }^{14,17}$. However, previous studies have reported some conflicting findings regarding the effect of sleep states on the cardiovascular changes during obstructive respiratory events, and not all studies have assessed BP changes during obstructive events in REM sleep ${ }^{18-20}$. Additionally, most previous studies did not account for obstructive respiratory events duration and the degree of desaturation on BP changes in different sleep stages. Therefore, further studies are needed to examine the effects of OSA on BP in REM and NREM sleep.

A non-invasive method that does not cause sleep disturbances is needed to assess BP continuously during different sleep stages to eliminate the effects of arousal and sleep disturbances on BP. Notably, a direct method based on the relationship between BP and the pulse wave velocity (PWV) can be used to determine BP noninvasively. Good correlations have been found between cardiovascular parameters and BP assessed using the pulse transit time (PTT) in patients with $\mathrm{OSA}^{21}$, and BP measured using the PTT is comparable with that assessed by reference methods ${ }^{22-25}$.

Because REM sleep is accompanied by a larger sympathetic surge and more accentuated hemodynamic fluctuations than in NREM sleep, the apnea-related rise in BP might be higher during REM sleep than during NREM sleep even if apnea duration oxygen desaturation were comparable. Therefore, we hypothesized that BP during and immediately after obstructive events is higher in REM sleep than in NREM sleep. To this end, this study non-invasively assessed BP during and immediately after REM and NREM obstructive events using the PTT-based method.

\section{Methods}

Subjects. This study recruited consecutive continuous positive airway pressure-naïve OSA patients (aged 18 years and above) who were diagnosed using overnight polysomnography (PSG) (SOMNOmedics GmbH, Randersacker, Germany) between January and July 2018. Patients with previous surgical procedures for treatment of snoring or OSA, daytime hypercapnia ( $\mathrm{PaCO} 2>45 \mathrm{mmHg}$ ), congestive heart failure, neuromuscular diseases, or home oxygen therapy were excluded from this study. Additionally, all subjects with arrhythmias, which may influence the electrocardiogram (ECG) and plethysmography signals and thereby impede an accurate detection of the PTT, were also excluded from the study. Initially, 44 OSA patients were recruited, but 12 patients were excluded because REM sleep was not achieved, or they did have REM sleep duration of $\geq 20$ min. Therefore, 32 patients with both NREM and REM sleep periods were included in this study.

The study was approved by the ethics committee of the College of Medicine at King Saud University, and written informed consent was obtained from all participants. The used methods were carried out in accordance with the Declaration of Helsinki and the relevant guidelines and regulations.

Sleep study. All participants underwent pulmonary function tests and arterial blood gases. Additionally, all undertook type I attended polysomnography, and the following physiological parameters were monitored: six leads of EEG (F4 - M1, C4 - M1, O2 - M1, F3 - M2, C3 - M2, and O1 - M2), electrooculography (EOG), chin electromyography (EMG), electrocardiography (ECG), oxygen saturation, chest and abdominal wall movements, air flow (thermistor and nasal pressure), and sleep position assessed using Somnoscreem plus ${ }^{\mathrm{TM}}$ diagnostic equipment (SOMNOscreen plus ${ }^{\mathrm{TM}}$, Randersacker, Germany).

Raw data were scored manually according to established scoring criteria ${ }^{26}$. Apnea was scored when a drop in the peak thermal sensor excursion of $\geq 90 \%$ of the baseline lasted for $\geq 10$ seconds. Hypopnea was considered when the nasal pressure signal decreased by $\geq 30 \%$ for at least $\geq 10$ seconds, resulting in a $\geq 3 \%$ decrease in oxygen saturation from the pre-event baseline or arousal. The AHI, which was computed by dividing the number of episodes of apneas and hypopneas by total sleep time in hours, is expressed as events/hour. The duration of the obstructive events was measured from the first breath that is clearly reduced to the beginning of the first breath that approximates the baseline amplitude ${ }^{26}$. Obstructive AHI was calculated during REM and NREM sleep. The desaturation index, which was assessed by dividing the number of desaturations (drop in oxygen of $\geq 3 \%$ ) by the total sleep time, is expressed as events per hour of sleep.

Arousal was scored during sleep stages N1, N2, N3, or REM when there was an abrupt shift of EEG frequency (including alpha, theta, and/or frequencies greater than $16 \mathrm{~Hz}$ [but not spindles]), which lasted at least 3 seconds, with at least 10 seconds of stable sleep preceding the change ${ }^{26}$. REM arousal scoring requires a concurrent increase in submental EMG lasting at least 1 second ${ }^{26}$.

Blood pressure measurement. BP was measured indirectly using a validated method based on the PTT. PWV and BP were assessed using the DOMINO-Software (DOMINO 2.2.0 supplied with the SOMNOscreen plus $^{\mathrm{TM}}$, Randersacker, Germany). ECG and the finger photoplethysmography curve were recorded with the SOMNOscreen $^{\mathrm{TM}}$ polysomnography device ${ }^{22,25,27}$. The SOMNOscreen plusTM noninvasive blood pressure measurement has been validated according to the European Society of Hypertension (ESH) protocol ${ }^{27}$.

SOMNOscreen plus ${ }^{\mathrm{TM}}$, which allows continuous (beat-to-beat) and noninvasive BP monitoring, includes a finger photoplethysmograph that additionally provides oxygen saturation measurement. Two bipolar ECG electrodes were attached parasternally at the second right and fifth left intercostal spaces. A ground electrode was fixed to the lower limb. The finger plethysmography curve was recorded with the SOMNOscreen ${ }^{\mathrm{TM}}$ polysomnography device 22,25 
$\mathrm{BP}$ was calculated using the DOMINO software with the one-point calibration based on a PWV-blood-pressure relation ${ }^{22,27}$. It has been demonstrated that the value obtained by this calibration remains constant ${ }^{22}$. Continuous BP estimation was based on the beat-to-beat determination of PTT, calculated as the interval between the ECG $\mathrm{R}$-waves and the detection of the corresponding pulse wave (revealed from the finger photoplethysmography signal) at the peripheral site.

SOMNOmedics uses the innovative PTT method for the determination of the blood pressure by means of a patented algorithm ${ }^{28}$. The calculation of the systolic and diastolic blood pressure is based on a non-linear correlation between blood pressure (in $\mathrm{mmHg}$ ) and PTT (in $\mathrm{ms})^{22}$. Systolic BP and diastolic BP readings were calculated based on the relationship between BP levels and $\mathrm{PTT}^{27}$.

$\mathrm{BP}$ was assessed during pre-obstructive events (10 sec of regular breathing pattern immediately preceding the obstructive event) (this will be called "Quiet Sleep" BP), obstructive events, and post-obstructive events (average of the 3 consecutive peak measurements within $15 \mathrm{sec}$ of event termination) ${ }^{29}$. Arterial pressure was measured on a beat-by-beat basis throughout the obstructive events and in the immediate post-obstructive event period during NREM and REM. The average values of BP during the pre-obstructive events, during obstructive events (both apneas and hypopneas), and in the immediate post-obstructive event period were used in the analysis. Additionally, BP was measured for $10 \mathrm{~min}$ while awake in the supine position in bed using the same method, and the average awake BP was also used in the analysis (this will be called "Awake" BP).

Statistical analysis. Data are expressed as means \pm SD for continuous data and $\mathrm{n}(\%)$ for categorical data. The student $\mathrm{t}$-test was used to compare continuous data if the normality assumption was met; otherwise, the Mann-Whitney U test was used. Analysis of variance was used for normally distributed data for comparison of three or more continuous variables; if the normality test failed, Kruskal-Wallis test by ranks was used. The $\chi 2$ test was used to compare categorical variables.

Simple linear regression was used to determine associations between changes in systolic BP during obstructive and post-obstructive events and independent factors. Independent variables included age, body mass index (BMI), desaturation index, arousal index, obstructive event duration, AHI, and history of hypertension.

Based on a previous study that assessed systolic BP changes after apnea ${ }^{30}$, and demonstrated a change in systolic BP of $8 \mathrm{mmHg}$ (SD 19.5) in REM sleep and a change of $6 \mathrm{mmHg}$ (SD 15.5) in NREM sleep, we estimated the needed sample size to detect similar differences as $n=27$ based on the following equation $\left(n=(Z \text {-score })^{2} \times\right.$ $\left.\mathrm{StdDev}^{2} /(\text { margin of error })^{2}\right)^{31}$.

A $P$ of $\leq 0.05$ was considered significant. Statistical analyses were performed using SPSS ver. 21.0 (Chicago, IL, USA).

Research involving human subjects. The study protocol was approved by the institutional review board at King Saud University, and informed consent was obtained from all the participants prior to inclusion in this study. The used methods were carried out in accordance with the Declaration of Helsinki and the relevant guidelines and regulations.

Informed consent. Written informed consent was obtained from all participants.

\section{Results}

Clinical and demographic characteristics of patients. The study included 32 patients (males: 50\%) with a mean age of $50.2 \pm 12.1$ years and a BMI of $34.1 \pm 7.9 \mathrm{~kg} / \mathrm{m}^{2}$. Table 1 shows the demographic and general information of the studied patients. Among the recruited patients, 10 (31.3\%) had hypertension. Table 2 shows the PSG findings of patients. The mean AHI was $40.1 \pm 27.6$ events/hr. AHI-NREM was $38.3 \pm 30.6$ events/hr, and AHI-REM was $51.9 \pm 28.3$ events/hr. BP was higher during wakefulness than during sleep; however, the difference did not reach statistical significance.

Blood pressure during the obstructive and post-obstructive events. Obstructive events in NREM and REM were available for analysis in all patients $(n=32)$. Figure 1 demonstrates a histogram of a patient showing an increase in BP during REM sleep with significant desaturation and Fig. 2 displays a zoomed epoch of 6 minutes during REM sleep showing an increase in blood pressure in the post-obstructive event period. REM, rapid eye movement.

Table 3 shows the mean systolic and diastolic BP assessed using the PTT method during wakefulness, sleep, REM and NREM obstructive respiratory events, and NREM and REM post-obstructive events. No statistically significant difference in BP (both systolic and diastolic) was detected between REM and NREM during obstructive events $(130.3 \pm 17 / 81.1 \pm 11.1 \mathrm{mmHg}$ versus $126.8 \pm 16.3 / 76.9 \pm 10.3 \mathrm{mmHg})$ or post-obstructive events $(130 \pm 17.4 / 80.8 \pm 11.4 \mathrm{mmHg}$ versus $127 \pm 15.5 / 78.3 \pm 9)$. Additionally, no differences were detected in obstructive respiratory event duration or time spent with $\mathrm{SPO}_{2}<90 \%$ between REM and NREM sleep ( $23.8 \pm 11.2 \mathrm{sec}$ versus $20.8 \pm 4.9 \mathrm{sec}$ and $13.8 \pm 19.4 \mathrm{~min}$ versus $11.9 \pm 22.0 \mathrm{~min}$, respectively).

Systolic BP increased by approximately $8 \mathrm{mmHg}$ and $4 \mathrm{mmHg}$ during obstructive events in REM and NREM events, respectively, compared with that in quiet sleep. Systolic BP increased by approximately $7 \mathrm{mmHg}$ and $4 \mathrm{mmHg}$ during the post-obstructive event period in REM and NREM sleep, respectively, compared with that in quiet sleep.

In a sub-group analysis, we analyzed BP data for patients without hypertension $(n=22)$ (Table 4$)$. As for the whole group, no statistically significant difference in BP (both systolic and diastolic) was detected between REM and NREM during obstructive events $(124.7 \pm 12.6 / 79.2 \pm 9.7 \mathrm{mmHg}$ versus $121.3 \pm 13.3 / 74.6 \pm 9.3 \mathrm{mmHg})$ or post-obstructive events $(121.2 \pm 13.2 / 79.7 \pm 9.8 \mathrm{mmHg}$ versus $117.6 \pm 13.3 / 74.7 \pm 9.5)$. Similarly, no differences were detected in obstructive respiratory event duration or time spent with $\mathrm{SPO}_{2}<90 \%$ between REM and NREM sleep. Systolic BP increased by approximately $7 \mathrm{mmHg}$ and $4 \mathrm{mmHg}$ during obstructive events in REM and 


\begin{tabular}{|c|c|}
\hline Variables & Mean \pm SD/n (\%) \\
\hline Age (years) & $50.2 \pm 12.1$ \\
\hline Body mass index $\left(\mathrm{kg} / \mathrm{m}^{2}\right)$ & $34.1 \pm 7.9$ \\
\hline Sex (male) & $16(50)$ \\
\hline Epworth Sleepiness Scale & $11.9 \pm 7$ \\
\hline Systolic BP (awake supine in bed) & $129 \pm 19.1$ \\
\hline Diastolic BP (awake supine in bed) & $76.6 \pm 11$ \\
\hline \multicolumn{2}{|l|}{ Arterial blood gases } \\
\hline $\mathrm{pH}$ & $7.4 \pm 0.1$ \\
\hline $\mathrm{PaCO}_{2}(\mathrm{mmHg})$ & $39.5 \pm 5.4$ \\
\hline $\mathrm{PaO}_{2}(\mathrm{mmHg})$ & $76.8 \pm 21.9$ \\
\hline $\mathrm{HCO}_{3}(\mathrm{mmol} / \mathrm{L})$ & $25.5 \pm 4.1$ \\
\hline $\mathrm{FEV}_{1} / \mathrm{FVC}(\%)$ & $88.8 \pm 7.8$ \\
\hline FVC (\% predicted) & $80.6 \pm 28$ \\
\hline $\mathrm{FEV}_{1}(\%$ predicted $)$ & $86.5 \pm 30.2$ \\
\hline \multicolumn{2}{|l|}{ Comorbidities } \\
\hline Hypertension & $10(31.3)$ \\
\hline Ischemic heart disease & $0(0)$ \\
\hline Diabetes mellitus & $9(29)$ \\
\hline Compensated heart failure & $0(0)$ \\
\hline Stroke & $0(0)$ \\
\hline Bronchial asthma & $3(9.4)$ \\
\hline Hypothyroidism & $5(15.6)$ \\
\hline Hypercholesterolemia & $10(31.3)$ \\
\hline \multicolumn{2}{|l|}{ OSA Severity } \\
\hline Mild OSA & $4(12.5)$ \\
\hline Moderate OSA & $12(37.5)$ \\
\hline Severe OSA & $16(50)$ \\
\hline
\end{tabular}

Table 1. Demographic and general information of patients. A total of 32 patients participated in this study. BP, blood pressure; OSA, obstructive sleep apnea; FVC, forced vital capacity; FEV, forced expiratory volume.

\begin{tabular}{|l|c|}
\hline Variables & Mean \pm SD/n (\%) \\
\hline Time in bed & $381.2 \pm 79.7$ \\
\hline Total sleep time & $290.5 \pm 65.4$ \\
\hline Sleep efficiency (\%) & $76 \pm 13.7$ \\
\hline Stage N1 (\%) & $8.3 \pm 7.3$ \\
\hline Stage N2 (\%) & $63.4 \pm 12.9$ \\
\hline Stage N3 (\%) & $13.7 \pm 13.2$ \\
\hline Stage REM (\%) & $13.4 \pm 5.5$ \\
\hline Apnea hypopnea index (AHI) (events/hr) & $40.1 \pm 27.6$ \\
\hline Obstructive apnea index (events/hr) & $3 \pm 6.1$ \\
\hline Obstructive hypopnea index (events/hr) & $35.9 \pm 23.6$ \\
\hline Desaturation index (desaturations/hr) & $23 \pm 22.8$ \\
\hline Time with SpO $2<90 \%(m i n s)$ & $12.8 \pm 17.8$ \\
\hline Lowest recorded SpO $(\%)$ & $82 \pm 10.5$ \\
\hline Mean nocturnal $\mathrm{SpO}_{2}(\%)$ & $94.1 \pm 2.8$ \\
\hline Arousal index (arousals/hr) & $33.2 \pm 20.1$ \\
\hline
\end{tabular}

Table 2. Polysomnographic findings of patients. A total of 32 patients participated in this study. REM, rapid eye movement; NREM, non-REM.

NREM events, respectively, compared with that in quiet sleep. Systolic BP increased by approximately $4 \mathrm{mmHg}$ during the post-obstructive event period in REM and no changes in systolic BP during NREM sleep, compared with that in quiet sleep.

Subsequently, we categorized patients into those with severe (AHI $\geq 30$ events/hr) and mild to moderate OSA $(\mathrm{AHI}<30$ events/hr) (Table 5). During NREM sleep obstructive events, patients with severe OSA (AHI $>30)$ had higher systolic pressure than did those with mild to moderate OSA $(130.1 \pm 17.3 \mathrm{mmHg}$ vs. $123.1 \pm 14.7 \mathrm{mmHg}$, $\mathrm{p}=0.05)$. However, during REM sleep obstructive events, no difference in BP was detected between patients 


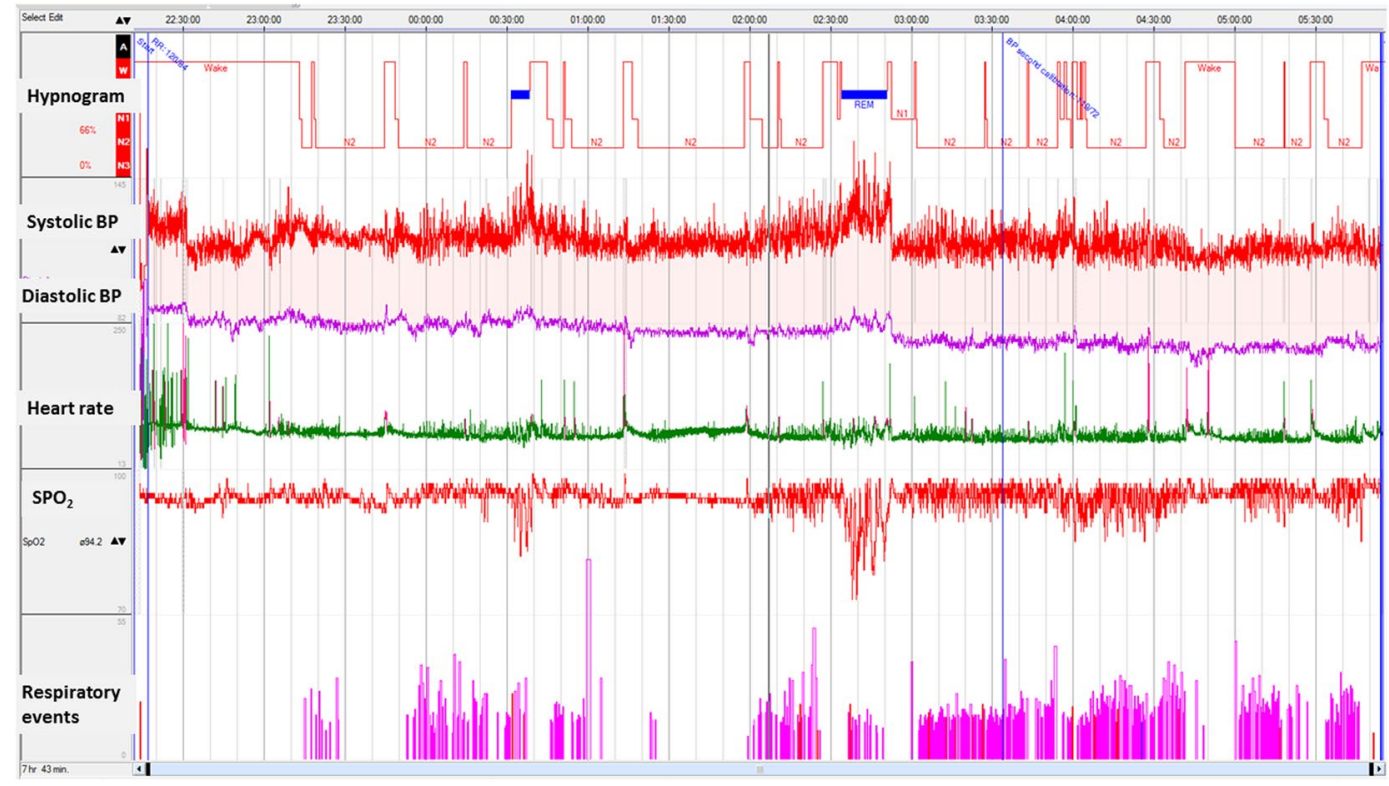

Figure 1. A histogram of a patient showing an increase in blood pressure during REM sleep with significant desaturation.

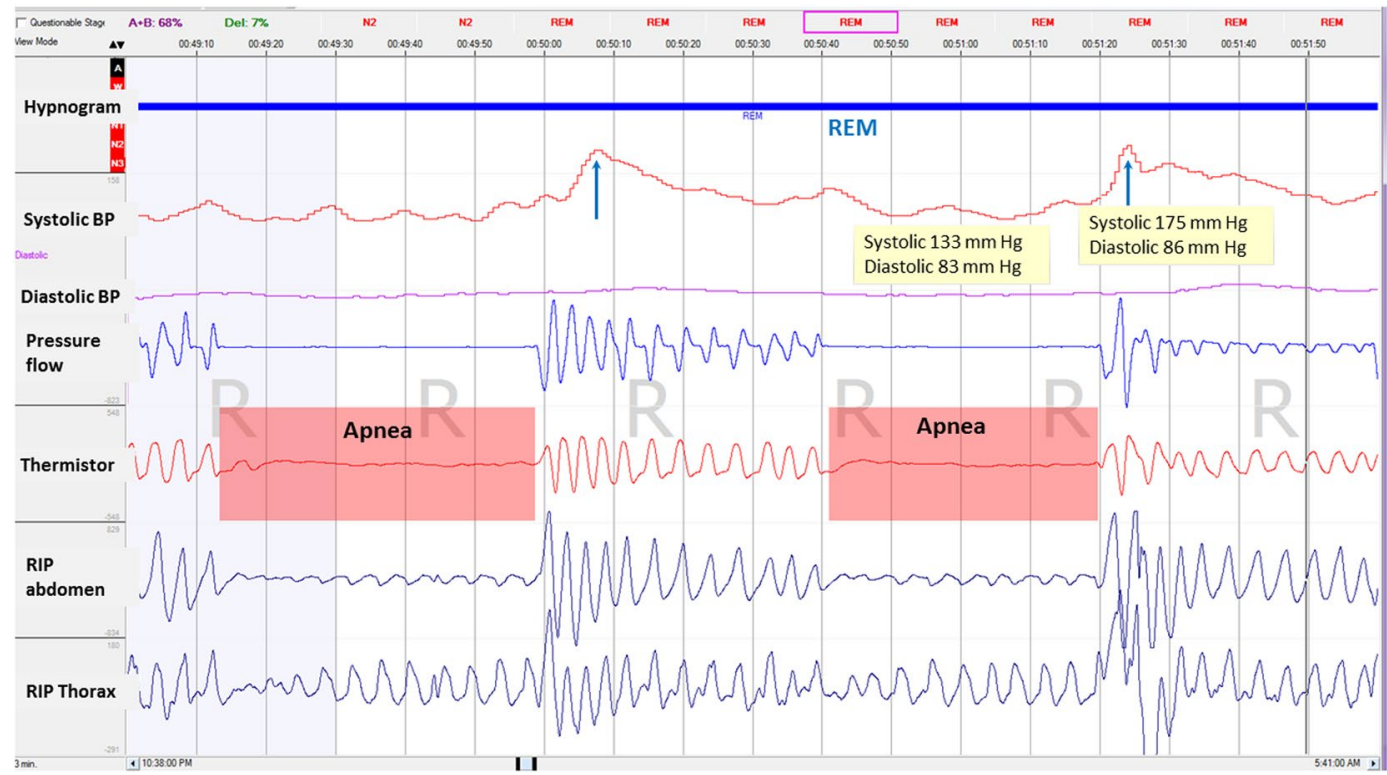

Figure 2. A zoomed epoch of 6 minutes during REM sleep showing an increase in blood pressure in the postobstructive event period (blue arrows). REM: rapid eye movement.

\begin{tabular}{|l|l|l|l|l|l|l|l|l|l|}
\hline \multirow{2}{*}{ Variable } & \multirow{3}{*}{} & \multirow{3}{*}{\begin{tabular}{l} 
During the obstructive event \\
\cline { 5 - 10 }
\end{tabular}} & Awake & Quiet Sleep & REM & NREM & Post-obstructive event \\
\hline Systolic BP & $129 \pm 19.1$ & $122.7 \pm 15.7$ & $130.3 \pm 17$ & $126.8 \pm 16.3$ & 0.4 & $130 \pm 17.4$ & $127 \pm 15.5$ & 0.3 \\
\hline Diastolic BP & $76.6 \pm 11$ & $76.8 \pm 10.4$ & $81.1 \pm 11.1$ & $76.9 \pm 10.3$ & 0.15 & $80.8 \pm 11.4$ & $78.3 \pm 9$ & 0.3 \\
\hline Mean apnea -hypopnea duration (Sec) & & & $23.8 \pm 11.2$ & $20.8 \pm 4.9$ & 0.6 & & & & \\
\hline Time with SpO2 $<90 \%$ (Min) & & & $13.8 \pm 19.4$ & $11.9 \pm 22.0$ & 0.7 & & & & \\
\hline
\end{tabular}

Table 3. Comparison of blood pressure during obstructive and post-obstructive respiratory events between REM and NREM sleep. 


\begin{tabular}{|l|l|l|l|l|l|l|l|l|l|}
\hline & & & & \multicolumn{5}{l|}{ During the obstructive event (n=22) } & \multicolumn{3}{l|}{ Post-obstructive event } \\
\cline { 5 - 10 } Variable & Awake & Quiet Sleep & REM & NREM & p-value & REM & NREM & p-value \\
\hline Systolic BP & $123.4 \pm 17.2$ & $117.6 \pm 13.3$ & $124.7 \pm 12.6$ & $121.3 \pm 13.3$ & 0.8 & $121.2 \pm 13.2$ & $117.6 \pm 13.3$ & 0.4 \\
\hline Diastolic BP & $74.4 \pm 10.2$ & $74.7 \pm 9.5$ & $79.2 \pm 9.7$ & $74.6 \pm 9.3$ & 0.2 & $79.7 \pm 9.8$ & $74.7 \pm 9.5$ & 0.1 \\
\hline $\begin{array}{l}\text { Mean apnea -hypopnea } \\
\text { duration (Sec) }\end{array}$ & & & $23.1 \pm 12.1$ & $19.9 \pm 6.1$ & 0.3 & & & \\
\hline Time with SpO2 $<90 \%$ (Min) & & & $12.9 \pm 19.4$ & $11.3 \pm 18.5$ & 0.7 & & & & \\
\hline
\end{tabular}

Table 4. Comparison of blood pressure during obstructive and post-obstructive respiratory events between REM and NREM sleep in patients without hypertension $(n=22)$.

\begin{tabular}{|c|c|c|c|c|c|c|c|c|}
\hline Variables & Awake & Sleep & $\begin{array}{l}\text { REM-OSA } \geq 30 \\
(\mathrm{n}=24)\end{array}$ & $\begin{array}{l}\text { REM-OSA }<30 \\
(n=8)\end{array}$ & p-value & $\begin{array}{l}\text { NREM-OSA } \geq 30 \\
(n=16)\end{array}$ & $\begin{array}{l}\text { NREM-OSA }<30 \\
(\mathrm{n}=16)\end{array}$ & p-value \\
\hline Mean obstructive event duration & $129 \pm 19.1$ & $122.7 \pm 15.7$ & $22.9 \pm 8.2$ & $26.2 \pm 18.1$ & 0.7 & $20.8 \pm 5.7$ & $20.9 \pm 4.1$ & 0.9 \\
\hline Time with SpO2 < 90\% (min) & $76.6 \pm 11$ & $76.8 \pm 10.4$ & $13.8 \pm 19.4$ & $7.3 \pm 4.5$ & 0.8 & $15.5 \pm 23.8$ & $2.7 \pm 4.1$ & 0.06 \\
\hline \multicolumn{9}{|l|}{ During the obstructive event } \\
\hline Systolic BP during obstructive event & & & $130.2 \pm 18.6$ & $130.7 \pm 12.3$ & 0.96 & $130.1 \pm 17.3$ & $123.1 \pm 14.7$ & 0.05 \\
\hline Diastolic BP during obstructive event & & & $82.6 \pm 10.2$ & $76.8 \pm 13$ & 0.2 & $79.9 \pm 11.7$ & $73.6 \pm 7.6$ & 0.3 \\
\hline \multicolumn{9}{|l|}{ Post-obstructive event } \\
\hline Systolic BP Post-Events & & & $129.4 \pm 19.2$ & $131.5 \pm 13.5$ & 0.8 & $131.8 \pm 17.8$ & $124.6 \pm 17.4$ & 0.4 \\
\hline Diastolic BP Post-Events & & & $81.9 \pm 10.6$ & $77.8 \pm 13.9$ & 0.4 & $82.8 \pm 10.7$ & $75.4 \pm 12.4$ & 0.1 \\
\hline
\end{tabular}

Table 5. Comparisons between patients with severe and mild to moderate OSA in REM and NREM sleep.

with mild to moderate OSA and those with severe OSA $(130.2 \pm 18.6 \mathrm{mmHg}$ vs. $130.7 \pm 12.3 \mathrm{mmHg})$. In the post-obstructive event period, no difference in BP was detected in both REM and NREM events between patients with severe and mild to moderate OSA.

Determinants of changes in blood pressure during REM and NREM obstructive events. Simple linear regression analysis for the prediction of systolic BP during obstructive events in REM and NREM is shown in Table 6. During obstructive events in REM sleep, oxygen desaturation index, awake BP, and hypertension were the significant predictors of large changes in systolic BP. However, during obstructive events in NREM, awake BP and hypertension were the only significant predictors of large changes in BP.

Simple linear regression analysis for the prediction of systolic BP during the post-obstructive event period in REM and NREM is shown in Table 7. During both REM and NREM sleep, history of hypertension and awake BP were the predictors of large changes in BP, and during REM sleep, oxygen desaturation index was a predictor of large changes in BP.

\section{Discussion}

This study assessed acute changes in BP during obstructive and post-obstructive respiratory events in adults. The findings revealed increased BP during obstructive events and post-obstructive event period in both REM and NREM sleep; however, the differences in BP between REM and NREM were not statistically significant during the obstructive events or the post-obstructive event period. Nevertheless, the obstructive event duration and degree of desaturation were similar in both REM and NREM sleep. Although the difference in BP did not reach statistical significance, systolic BP was higher by approximately $3.5 \mathrm{mmHg}$ in REM sleep compared to NREM sleep (Table 3), and it was sustained during post-obstructive event; the magnitude of the difference is clinically important though the lack of significance might be due to the relatively low sample size. Simple linear regression identified history of hypertension as a predictor of increased BP during obstructive events and the post-obstructive event period in both REM and NREM sleep.

Changes in BP during sleep in OSA patients are rapid with large swings; therefore, suitable methods are needed to monitor fast changes in BP, such as beat-by-beat measurement of BP. The PTT and PWV method is among the most common applications of beat-to-beat BP recordings $\mathrm{s}^{22,24,25,32}$. The beat-by-beat measurement of BP allows accurate detection of the very short-term variability in BP, although it may be less accurate in the assessment of absolute $\mathrm{BP}$ readings ${ }^{33}$.

Previous studies have demonstrated increased arterial BP following obstructive apnea in adult OSA patients ${ }^{18-20}$. Nevertheless, some conflicts exist in the reported data regarding the effect of sleep states on the cardiovascular changes during obstructive respiratory events, and not all studies have assessed BP changes during obstructive respiratory events in REM sleep. Jelic et al. found no differences in BP between sleep states; however, mean systolic BP was significantly lower during NREM apnea than during awake state but was significantly higher after apnea than during awake or apnea ${ }^{30}$. They also found that systolic BP changes during REM and NREM sleep had similar patterns with a reduction from the awake state to occurrence of apnea and an increase in the post-apnea period. In addition, Garpestad et al. demonstrated a higher BP elevation during post-obstructive events in both NREM and REM sleep, and the elevation was higher during REM sleep than during NREM sleep ${ }^{18}$. 


\begin{tabular}{|c|c|c|c|c|}
\hline \multicolumn{5}{|l|}{ During REM sleep obstructive events } \\
\hline Variables in the Equation & $\beta$ Coefficient & $\begin{array}{l}\text { Standard } \\
\text { Error }\end{array}$ & 95\% C.I. & P-Value \\
\hline Age (years) & 0.369 & 0.276 & $-0.199-0.937$ & 0.193 \\
\hline AHI-REM (events/hr) & -0.007 & 0.117 & $-0.249-0.234$ & 0.950 \\
\hline Desaturation Index (desaturations/hr) & 0.422 & 0.166 & $0.08-0.764$ & 0.018 \\
\hline Arousal Index (arousals/hr) & 0.243 & 0.158 & $-0.082-0.569$ & 0.136 \\
\hline Mean obstructive event duration (Seconds) & 0.427 & 0.705 & $-1.035-1.888$ & 0.551 \\
\hline Known hypertension & 16.839 & 6.210 & $4.049-29.629$ & 0.012 \\
\hline Awake blood pressure & 0.644 & 0.120 & $0.397-0.89$ & $<0.001$ \\
\hline Sex (Male) & 1.052 & 5.745 & $-10.698-12.802$ & 0.856 \\
\hline Diabetes mellitus & 4.854 & 6.172 & $-7.809-17.517$ & 0.438 \\
\hline \multicolumn{5}{|l|}{ During NREM sleep obstructive events } \\
\hline Age (years) & 0.403 & 0.242 & $-0.092-0.899$ & 0.106 \\
\hline AHI-NREM (events/hr) & 0.176 & 0.114 & $-0.059-0.411$ & 0.136 \\
\hline Desaturation Index (events/hr) & 0.254 & 0.166 & $-0.087-0.596$ & 0.138 \\
\hline Arousal Index (arousals/hr) & 0.062 & 0.147 & $-0.239-0.364$ & 0.674 \\
\hline Mean obstructive event duration (Seconds) & -0.673 & 0.618 & $-1.94-0.593$ & 0.285 \\
\hline Known hypertension & 18.438 & 5.600 & $6.968-29.908$ & 0.003 \\
\hline Awake blood pressure & 0.710 & 0.070 & $0.567-0.853$ & $<0.001$ \\
\hline Sex (Male) & 2.127 & 5.517 & $-9.141-13.394$ & 0.703 \\
\hline Diabetes mellitus & 6.246 & 6.155 & $-6.362-18.854$ & 0.319 \\
\hline
\end{tabular}

Table 6. Linear regression analysis to predict systolic blood pressure during REM and NREM sleep $(\mathrm{n}=32)$. (Enter Method).

\begin{tabular}{|c|c|c|c|c|}
\hline \multicolumn{5}{|l|}{ During post obstructive events arousal in REM sleep } \\
\hline Variables in the Equation & $\beta$ Coefficient & $\begin{array}{l}\text { Standard } \\
\text { Error }\end{array}$ & 95\% C.I. & P-Value \\
\hline Age (years) & 0.345 & 0.289 & $-0.25-0.94$ & 0.244 \\
\hline AHI-REM (events/hr) & -0.029 & 0.122 & $-0.28-0.222$ & 0.813 \\
\hline Desaturation Index (events/hr) & 0.440 & 0.173 & $0.084-0.796$ & 0.017 \\
\hline Arousal Index (arousals/hr) & 0.214 & 0.167 & $-0.13-0.557$ & 0.212 \\
\hline Mean obstructive event duration during REM (Seconds) & 0.246 & 0.724 & $-1.255-1.747$ & 0.737 \\
\hline Known hypertension & 17.761 & 6.441 & $4.495-31.027$ & 0.011 \\
\hline Awake blood pressure & 0.699 & 0.104 & $0.486-0.913$ & $<0.001$ \\
\hline Sex (Male) & 11.298 & 6.350 & $-1.754-24.351$ & 0.087 \\
\hline Diabetes mellitus & -1.710 & 7.841 & $-17.894-14.474$ & 0.829 \\
\hline \multicolumn{5}{|l|}{ During post obstructive events arousal in NREM sleep } \\
\hline Age (years) & 0.467 & 0.238 & $-0.02-0.954$ & 0.059 \\
\hline AHI-NREM (events/hr) & 0.116 & 0.117 & $-0.124-0.355$ & 0.330 \\
\hline Desaturation Index (events/hr) & 0.161 & 0.170 & $-0.187-0.51$ & 0.350 \\
\hline Arousal Index (arousals/hr) & -0.011 & 0.148 & $-0.313-0.292$ & 0.943 \\
\hline Mean obstructive event duration (Seconds)-NREM & -0.620 & 0.620 & $-1.891-0.651$ & 0.326 \\
\hline Known hypertension & 16.544 & 5.810 & $4.644-28.445$ & 0.008 \\
\hline Awake blood pressure & 0.705 & 0.074 & $0.552-0.857$ & $<0.001$ \\
\hline Sex (Male) & 5.643 & 5.577 & $-5.763-17.049$ & 0.320 \\
\hline Diabetes mellitus & 2.377 & 6.570 & $-11.104-15.859$ & 0.720 \\
\hline
\end{tabular}

Table 7. Linear regression analysis to predict systolic blood pressure during post-events arousal REM $(\mathrm{n}=32)$. (Enter Method).

Similarly, Okabe et al. showed a greater increase in BP in the post-obstructive event period during REM in adult OSA patients, and the obstructive events in REM sleep were longer and associated with greater oxygen desaturation than those in NREM sleep ${ }^{34}$. A later study in children with OSA (included only respiratory events $\geq 10 \mathrm{~s}$ in duration) also revealed that obstructive events were longer and associated with greater desaturation in REM sleep; however, the overall cardiovascular change was larger in $\mathrm{NREM}^{29}$. In the present study, BP was higher during obstructive events and post-obstructive event period in both REM and NREM sleep than in quiet sleep, although this difference did not reach statistical significance. 
Several mechanisms have been proposed to explain the increase in BP during or after an obstructive respiratory event. The obstructive event during sleep is associated with increased negative intrathoracic pressure, hypoxia, hypercapnia, and arousal responses, and these changes induce drastic increases in sympathetic activity ${ }^{35}$. During an obstructive event, the inhibitory effect of the thoracic afferents is absent, thus potentiating sympathetic activation ${ }^{36}$. As a result, vasoconstriction develops and causes a significant elevation in BP. At the end of the obstructive event, breathing resumes and apnea-induced restrictions on stroke volume and heart rate are abruptly eliminated, allowing ejection of the augmented cardiac output into a peripheral vascular bed that has been restricted by the sympathetic induce vasoconstriction; thus, a transient increase in BP might be induced in the immediate post-apnea period accordingly ${ }^{7,36}$.

Sleep stage may also affect BP in patients with OSA because the duration and severity of obstructive events vary with sleep stages. Obstructive events are less frequent during slow-wave sleep, and obstructive respiratory events are usually longer and associated with worse hypoxemia during REM sleep ${ }^{37,38}$. However, no significant difference was detected in BP during obstructive events between REM and NREM sleep in this study (Table 3). This could be related to the fact that no differences in the duration of obstructive events or time spent with $\mathrm{SPO}_{2}<90 \%$ between REM and NREM sleep were detected in the present study. A previous study assessed the relationship between obstructive event duration and concomitant BP changes in nine patients with OSA ${ }^{39}$. As the duration of the obstructive event increased from $10 \mathrm{sec}$ to greater than $30 \mathrm{sec}, \mathrm{BP}$ increased ${ }^{39}$. Another study demonstrated that OSA patients with a longer obstructive event duration had more severe hypertension when compared with patients with a shorter event duration ${ }^{40}$. Garpestad et al. reported that during REM sleep, prolongation of apnea duration resulted in lower oxygen desaturation and was associated with further increases in post-apneic $\mathrm{BP}^{18}$. Another interesting finding of this study is BP response during obstructive events in REM sleep. BP was similar in mild-to moderate and severe OSA in REM sleep, while BP response was lower in mild-to-moderate OSA in NREM sleep. This finding may mean that BP increase starts already with lower degree of OSA during REM sleep while the changes are notable first in severe OSA in NREM sleep. Further studies are needed to confirm this finding.

A plausible explanation for the reported relationship between BP and obstructive event duration is that, the longer the duration of the event, the higher the hypercapnia and the lower the hypoxemia ${ }^{39}$. Hypercapnia and hypoxemia synergistically act to increase sympathetic nerve activity ${ }^{41}$. The current study findings that oxygen desaturation was a predictor of increased BP during REM sleep obstructive events concur with the above explanation. Cyclical changes in BP have been observed during repeated intermittent hypoxemia in awake patients with $\mathrm{OSA}^{34}$. Moreover, increased ventilatory response to hypoxia may lead to a larger inspiratory effort upon resumption of breathing, which could result in increased $\mathrm{BP}^{29,34}$.

This study has some strengths. First, the study included patients with both REM and NREM obstructive events, thus allowing the comparison between REM and NREM sleep in the whole study group. Second, the study used a well-validated noninvasive and continuous beat-to-beat measurement method. However, this study does have several limitations. First, although we calculated the sample size, the studied sample remains relatively small. Therefore, studies with a larger sample size are needed to allow better detection of differences in BP. Second, the current findings do not agree with our hypothesis and cannot explain why REM-OSA is associated with high hypertension. This could be related to the fact that mean obstructive respiratory events duration and the degree of oxygen desaturation were not different in both REM and NREM respiratory events. Additionally, the current findings challenge recent data showing that REM-OSA is the main culprit for hypertension in patients with OSA, which calls for more research to verify the association between REM-OSA and hypertension. Third, the current paper has no control group. Therefore, future studies should include hypertensive patients with and without OSA.

In summary, this study revealed increased BP during obstructive events and in the post-obstructive event period in both REM and NREM sleep. However, no statistically significant differences in BP between REM and NREM sleep were detected. The lack of difference in BP between obstructive events in REM and NREM sleep could be related to the fact that obstructive event duration and the degree of desaturation were comparable in REM and NREM obstructive events in the study group. Future studies should assess the relationship between apnea duration in REM and NREM sleep and changes of BP as well as prevalent and incident hypertension.

Simple linear regression identified history of hypertension as a predictor of increased BP during obstructive events and in the post-obstructive event period in both REM and NREM sleep. Further studies with non-intrusive technologies are needed to enable frequent and accurate assessments of vascular function during different stages.

Received: 13 December 2019; Accepted: 10 February 2020;

Published online: 24 February 2020

\section{References}

1. Köseoğlu, H. I., Kanbay, A. \& Demir, O. A Different Clinical Type of OSAS: REM-Related OSAS. Eurasian J. Pulmonol. 17, 92-97 (2015).

2. Almeneessier, A. S. et al. Long-term adherence to continuous positive airway pressure in patients with rapid eye movement-only obstructive sleep apnea: a prospective cohort study. J. Thorac. Dis. 9, 3755-3765, https://doi.org/10.21037/jtd.2017.09.57 (2017)

3. Subramani, Y. et al. Understanding Phenotypes of Obstructive Sleep Apnea: Applications in Anesthesia, Surgery, and Perioperative Medicine. Anesth. Analg. 124, 179-191, https://doi.org/10.1213/ANE.0000000000001546 (2017).

4. Somers, V. K., Dyken, M. E., Mark, A. L. \& Abboud, F. M. Sympathetic-nerve activity during sleep in normal subjects. N. Engl. J. Med. 328, 303-307, https://doi.org/10.1056/NEJM199302043280502 (1993).

5. Mokhlesi, B. et al. Obstructive sleep apnea during REM sleep and hypertension. results of the Wisconsin Sleep Cohort. Am. J. Respir. Crit. Care Med. 190, 1158-1167, https://doi.org/10.1164/rccm.201406-1136OC (2014).

6. Mokhlesi, B. et al. Obstructive sleep apnoea during REM sleep and incident non-dipping of nocturnal blood pressure: a longitudinal analysis of the Wisconsin Sleep Cohort. Thorax 70, 1062-1069, https://doi.org/10.1136/thoraxjnl-2015-207231 (2015).

7. Lanfranchi, P. A., Pépin, J. L. \& Somers, V. K. In Principles and Practice of Sleep Medicine (eds M.H. Kryger \& T. Roth) Ch. 14, 142154 (Elsevier, Inc., (2017). 
8. Imadojemu, V. A., Gleeson, K., Gray, K. S., Sinoway, L. I. \& Leuenberger, U. A. Obstructive apnea during sleep is associated with peripheral vasoconstriction. Am. J. Respir. Crit. Care Med. 165, 61-66, https://doi.org/10.1164/ajrccm.165.1.2009062 (2002).

9. Tun, Y. et al. Nocturnal blood pressure during apnoeic and ventilatory periods in patients with obstructive sleep apnoea. Eur. Respir. J. 14, 1271-1277 (1999).

10. Planes, C. et al. Exacerbation of sleep-apnoea related nocturnal blood-pressure fluctuations in hypertensive subjects. Eur. Respir. J. 20, 151-157 (2002)

11. Davies, R. J., Crosby, J., Vardi-Visy, K., Clarke, M. \& Stradling, J. R. Non-invasive beat to beat arterial blood pressure during nonREM sleep in obstructive sleep apnoea and snoring. Thorax 49, 335-339 (1994).

12. Leroy, M. et al. Short-term variability of blood pressure during sleep in snorers with or without apnea. Hypertension 28, 937-943 (1996).

13. Marrone, O., Salvaggio, A., Bonsignore, M. R., Insalaco, G. \& Bonsignore, G. Blood pressure responsiveness to obstructive events during sleep after chronic CPAP. Eur. Respir. J. 21, 509-514 (2003).

14. Sforza, E., Capecchi, V. \& Lugaresi, E. Haemodynamic effects of short-term nasal continuous positive airway pressure therapy in sleep apnoea syndrome: monitoring by a finger arterial pressure device. Eur. Respir. J. 5, 858-863 (1992).

15. Sforza, E. \& Lugaresi, E. Determinants of the awakening rise in systemic blood pressure in obstructive sleep apnea syndrome. Blood Press. 4, 218-225 (1995)

16. Coccagna, G., Mantovani, M., Brignani, F., Parchi, C. \& Lugaresi, E. Continuous recording of the pulmonary and systemic arterial pressure during sleep in syndromes of hypersomnia with periodic breathing. Bull. Physiopathol. Respir. 8, 1159-1172 (1972).

17. Grote, L. et al. Assessment of the nocturnal blood pressure relative to sleep stages in patients with obstructive sleep apnea. Z. Kardiol. 85(Suppl 3), 112-114 (1996).

18. Garpestad, E., Ringler, J., Parker, J. A., Remsburg, S. \& Weiss, J. W. Sleep stage influences the hemodynamic response to obstructive apneas. Am. J. Respir. Crit. Care Med. 152, 199-203, https://doi.org/10.1164/ajrccm.152.1.7599824 (1995).

19. Garpestad, E. et al. Stroke volume and cardiac output decrease at termination of obstructive apneas. J. Appl. Physiol. 73, 1743-1748, https://doi.org/10.1152/jappl.1992.73.5.1743 (1992)

20. Ali, N. J., Davies, R. J., Fleetham, J. A. \& Stradling, J. R. The acute effects of continuous positive airway pressure and oxygen administration on blood pressure during obstructive sleep apnea. Chest 101, 1526-1532 (1992).

21. Pitson, D. J. \& Stradling, J. R. Value of beat-to-beat blood pressure changes, detected by pulse transit time, in the management of the obstructive sleep apnoea/hypopnoea syndrome. Eur. Respir. J. 12, 685-692 (1998).

22. Gesche, H., Grosskurth, D., Kuchler, G. \& Patzak, A. Continuous blood pressure measurement by using the pulse transit time: comparison to a cuff-based method. Eur. J. Appl. Physiol. 112, 309-315, https://doi.org/10.1007/s00421-011-1983-3 (2012).

23. Bartsch, S. et al. [Validation of continuous blood pressure measurements by pulse transit time: a comparison with invasive measurements in a cardiac intensive care unit]. Dtsch. Med. Wochenschr. 135, 2406-2412, https://doi.org/10.1055/s-0030-1269408 (2010).

24. Schmalgemeier, H. et al. Pulse transit time: validation of blood pressure measurement under positive airway pressure ventilation. Sleep. Breath. 16, 1105-1112, https://doi.org/10.1007/s11325-011-0609-7 (2012).

25. Patzak, A., Mendoza, Y., Gesche, H. \& Konermann, M. Continuous blood pressure measurement using the pulse transit time: Comparison to intra-arterial measurement. Blood Press. 24, 217-221, https://doi.org/10.3109/08037051.2015.1030901 (2015).

26. Berry, R. B. et al. AASM Scoring Manual Updates for 2017 (Version 2.4). J. Clin. Sleep. Med. 13, 665-666, https://doi.org/10.5664/ jcsm.6576 (2017).

27. Bilo, G. et al. Validation of the Somnotouch-NIBP noninvasive continuous blood pressure monitor according to the European Society of Hypertension International Protocol revision 2010. Blood Press. Monit. 20, 291-294, https://doi.org/10.1097/ MBP.0000000000000124 (2015).

28. Kuchler, G. Noninvasive blood pressure determination method and apparatus. US patent US007374542B2 (2008).

29. O'Driscoll, D. M. et al. Acute cardiovascular changes with obstructive events in children with sleep disordered breathing. Sleep. 32, 1265-1271 (2009).

30. Jelic, S. et al. Arterial stiffness increases during obstructive sleep apneas. Sleep. 25, 850-855 (2002).

31. Charan, J. \& Biswas, T. How to calculate sample size for different study designs in medical research? Indian. J. Psychol. Med. 35, 121-126, https://doi.org/10.4103/0253-7176.116232 (2013).

32. Gomez Garcia, M. T. et al. Can pulse transit time be useful for detecting hypertension in patients in a sleep unit? Arch. Bronconeumol. 50, 278-284, https://doi.org/10.1016/j.arbres.2013.12.001 (2014).

33. Marrone, O. \& Bonsignore, M. R. Blood-pressure variability in patients with obstructive sleep apnea: current perspectives. Nat. Sci. Sleep. 10, 229-242, https://doi.org/10.2147/NSS.S148543 (2018).

34. Okabe, S. et al. Role of hypoxia on increased blood pressure in patients with obstructive sleep apnoea. Thorax 50, 28-34 (1995).

35. Sei, H. Blood pressure surges in REM sleep: A mini review. Pathophysiology 19, 233-241, https://doi.org/10.1016/j. pathophys.2012.04.006 (2012).

36. Dempsey, J. A., Veasey, S. C., Morgan, B. J. \& O’Donnell, C. P. Pathophysiology of sleep apnea. Physiol. Rev. 90, 47-112, https://doi. org/10.1152/physrev.00043.2008 (2010).

37. Findley, L. J., Wilhoit, S. C. \& Suratt, P. M. Apnea duration and hypoxemia during REM sleep in patients with obstructive sleep apnea. Chest 87, 432-436 (1985).

38. Ratnavadivel, R. et al. Marked reduction in obstructive sleep apnea severity in slow wave sleep. J. Clin. Sleep. Med. 5, 519-524 (2009).

39. Alex, R. et al. Effect of apnea duration on apnea induced variations in cerebral blood flow velocity and arterial blood pressure. Conf. Proc. IEEE Eng. Med. Biol. Soc. 2014, 270-273, https://doi.org/10.1109/EMBC.2014.6943581 (2014).

40. Wu, H., Zhan, X., Zhao, M. \& Wei, Y. Mean apnea-hypopnea duration (but not apnea-hypopnea index) is associated with worse hypertension in patients with obstructive sleep apnea. Med. 95, e5493, https://doi.org/10.1097/MD.0000000000005493 (2016).

41. Somers, V. K., Mark, A. L. \& Abboud, F. M. Sympathetic activation by hypoxia and hypercapnia-implications for sleep apnea. Clin. Exp. Hypertens. A. 10(Suppl 1), 413-422 (1988).

\section{Acknowledgements}

This work was supported by the Strategic Technologies Program of the National Plan for Sciences and Technology and Innovation in the Kingdom of Saudi Arabia (MED511-02-08).

\section{Author contributions}

A.S.A.: Contributions to the conception and design of the work; interpretation of the data, writing of the manuscript, agreed both to be personally accountable for the author's own contributions and to ensure that questions related to the accuracy or integrity of any part of the work, even ones in which the author was not personally involved. M.A. and S.A.: Contributions to the conception and design of the work; writing the manuscript; and final approval of the version to be submitted, agreed both to be personally accountable for the author's own contributions and to ensure that questions related to the accuracy or integrity of any part of the work, even ones in which the author was not personally involved. O.S.H.: Data cleaning; writing of the manuscript 
and final approval of the version to be submitted, agreed both to be personally accountable for the author's own contributions and to ensure that questions related to the accuracy or integrity of any part of the work, even ones in which the author was not personally involved. A.H.O.: Data cleaning, interpretation of the data, statistical analysis; writing of the manuscript and final approval of the version to be submitted, agreed both to be personally accountable for the author's own contributions and to ensure that questions related to the accuracy or integrity of any part of the work, even ones in which the author was not personally involved. A.S.B.: Conception and design of the work; data acquisition, analysis, and interpretation; securing funds; writing the manuscript; and final approval of the version to be submitted, agreed both to be personally accountable for the author's own contributions and to ensure that questions related to the accuracy or integrity of any part of the work, even ones in which the author was not personally involved.

\title{
Competing interests
}

The authors declare no competing interests.

\section{Additional information}

Correspondence and requests for materials should be addressed to A.S.A. or A.S.B.

Reprints and permissions information is available at www.nature.com/reprints.

Publisher's note Springer Nature remains neutral with regard to jurisdictional claims in published maps and institutional affiliations.

\begin{abstract}
(c) (i) Open Access This article is licensed under a Creative Commons Attribution 4.0 International License, which permits use, sharing, adaptation, distribution and reproduction in any medium or format, as long as you give appropriate credit to the original author(s) and the source, provide a link to the Creative Commons license, and indicate if changes were made. The images or other third party material in this article are included in the article's Creative Commons license, unless indicated otherwise in a credit line to the material. If material is not included in the article's Creative Commons license and your intended use is not permitted by statutory regulation or exceeds the permitted use, you will need to obtain permission directly from the copyright holder. To view a copy of this license, visit http://creativecommons.org/licenses/by/4.0/.
\end{abstract}

(C) The Author(s) 2020 